\title{
The recognition of HIV-1 consensus group M Gag and Nef peptide reagents in mono- and multi-clade epidemics: implications for HIV vaccine design
}

\author{
L Zembe $^{1 *}$, M Tongo $^{2}$, E Ebong $^{3}$, EM Ngobe ${ }^{3}$, C Williamson $^{2}$, W Burgers ${ }^{2}$
}

From AIDS Vaccine 2012

Boston, MA, USA. 9-12 September 2012

\section{Background}

The high level of genetic diversity of HIV-1 poses a major challenge for global vaccine development. Vaccines based on centralized sequences would minimize genetic distances to multiple clades and potentially maximize crossreactivity. Whether reactivity of these centralized peptide reagents differs in mono- and multi-clade epidemic is unknown.

\section{Methods}

In this study, full-length gag and nef gene sequences (from Cameroon, 50 and 54, respectively and South Africa, 23 and 19, respectively) were characterized. HIV-specific $\mathrm{T}$-cell responses to group $\mathrm{M}$ consensus Gag and Nef peptide reagents were characterized at the peptide level using the IFN- $\gamma$ ELISpot assay.

\section{Results}

Viruses from Cameron exhibited a large degree of genetic diversity; all subtypes were present excluding subtype $C$, with CRF02_AG being dominating (49\%). Contrary, sequenced viruses from South Africa were all pure subtype $\mathrm{C}$ viruses. Despite the greater diversity of viral clades in the Cameroonian cohort, the genetic distances to the consensus $M$ reagents was similar for both cohorts $(11 \%$ and $15 \%$ for Gag and Nef, respectively). Whilst the magnitude and breadth of responses to Con M Gag and Nef did not differ significantly between the two cohorts, there was a greater frequency of responders in the Cameroonian cohort compared to South Africans (95\% versus 82\%, respectively). For the Cameroonian cohort, 75/182 (41\%) of the consensus $M$ peptides were targeted, while for the South African cohort 66/182 (36\%) of the peptides were targeted, with the majority being recognized in only 1-2 participants (69\% and $76 \%$, respectively). Patterns of immunodominance and targeting, however, differed dramatically between the two cohorts, with only $36 \%$ and $39 \%$ of Gag and Nef peptides commonly recognized between the two cohorts.

\section{Conclusion}

Although similarities in total magnitude and breadth may be observed between different epidemics, patterns of immunodominance of centralized immunogens may be different which may have implications for vaccine development.

\section{Author details}

${ }^{1}$ University of Cape Town, Cape Town, South Africa. ${ }^{2}$ Division of Virology, University of Cape Town, Cape Town, South Africa. ${ }^{3}$ Institute of Medical Research and Study of Medicinal Plants, Younde, Cameroon.

Published: 13 September 2012

doi:10.1186/1742-4690-9-S2-P253

Cite this article as: Zembe et al:: The recognition of HIV-1 consensus group M Gag and Nef peptide reagents in mono- and multi-clade epidemics: implications for HIV vaccine design. Retrovirology 2012 9(Suppl 2):P253. 